\title{
Fragebogen zu psychologischen Einflussfaktoren der Nutzung von Pkw, ÖPNV und Fahrrad (PsyVKN)
}

\author{
Faktorenstruktur, psychometrische Eigenschaften und \\ Validierung
}

\author{
Marcel Hunecke ${ }^{1}$, Holger Heppner ${ }^{1}$ und Sören Groth ${ }^{1,2}$ \\ 'Fachhochschule Dortmund \\ 2ILS - Institut für Landes- und Stadtentwicklungsforschung, Dortmund
}

\begin{abstract}
Zusammenfassung: Zur Analyse der Ursachen für die interindividuelle Variabilität der Verkehrsmittelnutzung werden subjektive Bewertungen von Verkehrsmitteln im Rahmen standardisierter Verkehrserhebungen zunehmend mit berücksichtigt. Im vorliegenden Beitrag wird ein theoretisch fundierter und empirisch validierter Fragebogen zu den psychologischen Einflussfaktoren der Nutzung von Pkw, ÖPNV und Fahrrad (PsyVKN) im Alltag vorgestellt, der Items zu Kontrollüberzeugungen, verkehrsmittelbezogenen Normen und Einstellungen umfasst. Auf der empirischen Basis von sechs Studien mit 10850 Befragten aus über neun Jahren psychologischer Mobilitätsforschung wurde, auf der Basis von Strukturgleichungsmodellen und Messinvarianzanalysen, ein ökonomisches, reliables und valides Messinstrument für psychologische Merkmale der Verkehrsmittelnutzung entwickelt. Als Ergebnis stehen acht psychologische Konstrukte mit 21 Items für die Anwendung in der interdisziplinären Verkehrs- und Mobilitätsforschung zur Verfügung. Die entsprechenden psychologischen Konstrukte können in Verkehrserhebungen zur Erfassung der interindividuellen Variabilität bei der Verkehrsmittelnutzung und darauf aufbauend zur Planung von Maßnahmen zur Reduktion der Automobilität im Nahverkehr eingesetzt werden.
\end{abstract}

Schlüsselwörter: verkehrsmittelbezogene Einstellungen, Normen, Überzeugungen, Messinvarianz, SEM

A Psychological Evaluation of a Questionnaire on use of Travel Modes. Factor Structure, Psychometric Properties, and Validation

Abstract: Subjective evaluations of different transport modes are often included in standardized transport surveys to analyze the reasons for interindividual variability in the use of travel mode. This paper presents a theoretically based and empirically validated questionnaire on the psychological determinants of choosing car, public transport, and bicycle transportation (PsyVKN), which includes items on control beliefs as well as norms and attitudes related to the respective transport mode. On the empirical basis of six studies with 10,850 participants from more than nine years of psychological mobility research, we developed an economic, reliable, and valid measurement instrument for the psychological characteristics of transport use, based on structural equation models and measurement invariance analyses. This results in eight psychological constructs, operationalized by 21 items, that are now available for future application in interdisciplinary mobility research. These psychological constructs can be employed in transport surveys to evaluate the interindividual variability in the use of travel mode and, subsequently, to plan interventions for reducing car use.

Keywords: transport-related attitudes, norms, beliefs, travel mode choice, measurement invariance, SEM

Räumliche Mobilität ermöglicht Individuen in modernen Gesellschaften die außerhäusliche Teilnahme am Erwerbsleben, an Freizeitaktivitäten, die Wahrnehmung sozialer Kontakte sowie die Versorgung mit Gütern und Dienstleistungen mittels der dafür erforderlichen Verkehrsmittel. Dabei lässt sich in den vergangenen Jahrzehnten in der Gesamtbevölkerung eine weitgehend konstante Verkehrsnachfrage beobachten, die durch ein weitgehend konstantes Verkehrsaufkommen pro Person und
Tag bei gleichzeitiger Zunahme des motorisierten Individualverkehrs, der Reisegeschwindigkeiten und der Distanzen jeweils zulasten anderer Verkehrsmittel beschrieben wird (Bundesministerium für Verkehr und digitale Infrastruktur [BMVI], 2019). Auf einer disaggregierten Ebene zeigt sich jedoch eine hohe interindividuelle Variabilität in der Verkehrsmittelnutzung, die nicht nur mit räumlichen Merkmalen und spezifischen sozialen Lebenslagen, sondern auch mit individuellen Bewertungen hinsichtlich 
der Nutzung unterschiedlicher Verkehrsmittel zusammenhängt (Ecke et al., 2019; Institut für angewandte Sozialwissenschaft GmbH [Infas], 2018).

Für die interdisziplinär ausgerichtete Verkehrs- und Mobilitätsforschung stellt die Berücksichtigung interindividueller Variabilität bei der Verkehrsmittelnutzung eine theoretische Erweiterung dar, die zu einer vollständigeren Erklärung der Verkehrsgenese dienen kann (Scheiner, 2016). Hierbei wird angestrebt, das realisierte Verkehrsverhalten in seinen komplexen wechselseitigen Bezügen aus verkehrs- und raumstrukturellen, organisatorischen und individuellen Einflussfaktoren möglichst umfassend zu erklären. Hierauf aufbauend können dann Handlungsund Gestaltungsempfehlungen für eine umwelt- und sozialverträgliche Verkehrsplanung abgeleitet werden (BuschGeertsema, Klinger \& Lanzendorf, 2015).

Im vorliegenden Artikel wird mit dem Fragebogen zur subjektiven Bewertung der nahräumlichen Nutzung von Pkw, ÖPNV und Fahrrad (PsyVKN) ein Messinstrument vorgestellt, das verkehrsmittelbezogene Einstellungen, Kontrollüberzeugungen und Normen umfasst, die sich durch Zusammenhänge zur individuellen Verkehrsmittelnutzung auszeichnen. Der PsyVKN basiert auf einer breiten empirischen Basis von sechs Studien mit 10850 befragten Personen, die in einem Zeitraum von neun Jahren durchgeführt wurden. Der PsyVKN lässt sich in standardisierten Verkehrsbefragungen einsetzen und ermöglicht so vertiefende Analysen zum Zusammenwirken von Personen- und Umweltmerkmalen bei der Verkehrsmittelnutzung.

\section{Psychologie der Verkehrsmittelnutzung}

Aus psychologischer Perspektive wird die Verkehrsmittelnutzung auf der Grundlage von Konstrukten aus empirisch fundierten Handlungstheorien erklärt (Bamberg \& Schmidt, 2003). Hierbei haben sich vor allem die Theory of Planned Behaviour (TPB) von Ajzen (1991), z.B. bei Bamberg, Ajzen und Schmidt (2003), und das Norm-Aktivations-Modell (NAM) von Schwartz (1977), z. B. bei Nordlund und Garvill (2003), empirisch bewährt. Während die TPB die zentralen psychologischen Einflussfaktoren für entscheidungsbasiertes Verhalten erfasst, bezieht sich der Erklärungsanspruch des NAM auf Verhalten, das stark durch Normen bestimmt wird, wie prosoziales oder ökologisches Verhalten. In ihrer Anwendung auf die Verkehrsmittelnutzung ergänzen sich die Konstrukte aus beiden Handlungstheorien und lassen sich in einen integrierten Erklärungsansatz zusammenführen, der sich als eine erweiterte Theorie des geplanten Verhaltens charakterisieren lässt (Heath \& Gifford, 2002; Bamberg, Hunecke \& Blöbaum, 2007; Lo, Van Breukelen, Peters \& Kok, 2016).
In dem integrierten Erklärungsansatz kommt den Konstrukten Intention, Einstellung, subjektive Norm und wahrgenommene Verhaltenskontrolle aus der TPB eine zentrale Bedeutung zu, die vor allem durch das Konstrukt der personalen Norm aus der NAM ergänzt wird. Die Relevanz der handlungstheoretischen Konstrukte wahrgenommene Verhaltenskontrolle, subjektive Normen, Einstellungen und personaler Normen wird ebenfalls durch MetaAnalysen zur Nutzung von Pkws und von Bus und Bahn im Nahverkehr bestätigt (Gardner \& Abraham, 2008; Lanzini \& Khan, 2017; Hoffmann, Abraham, White, Ball \& Skippon, 2017). Die vorliegenden empirischen Befunde zur Verkehrsmittelnutzung beziehen sich weitgehend auf den Nahverkehr und nicht auf die Nutzung von Verkehrsmitteln im Fernverkehr, wie Fernzüge und Flugzeuge. Weiterhin werden Verhaltensgewohnheiten (Habits), für die sich ebenfalls konsistente Zusammenhänge zur Verkehrsmittelwahl nachweisen lassen (Gardner, 2009), von den weiteren Betrachtungen ausgeklammert, weil diese durch ihren automatisierten Funktionsmodus nicht an intendierten Prozessen der Verkehrsmittelnutzung beteiligt sind. Ebenso werden subjektive Bewertungen in Bezug auf das Zufußgehen nicht berücksichtigt, weil zu diesem Modus der Verkehrsmittelnutzung noch keine ausreichend abgesicherten Befunde vorliegen.

Die zur Erklärung der Verkehrsmittelnutzung im Nahraum relevanten handlungstheoretischen Konstrukte lassen sich drei Klassen von psychologischen Einflussfaktoren zuordnen: Normen, Kontrollüberzeugungen und Einstellungen.

Normen verweisen auf Anweisungen und Verpflichtungen $\mathrm{zu}$ angemessenem und unangemessenem Verhalten. In der Metaanalyse von Gardner und Abraham (2008) zeigt sich für die personale und soziale Norm hinsichtlich einer Nichtnutzung des Pkw eine negative Korrelation zur Pkw-Nutzung von $r=-.41$ und $r=-.36$. Soziale Normen wie sie etwa im Rahmen der TPB als subjektive Normen bezeichnet werden - beziehen sich auf die Erwartungshaltung von sozialen Gruppen, die für die eigene Person als relevant erachtet werden. Im Rahmen der Alltagsmobilität kommen diese vor allem im Hinblick auf den sozialen Status der Verkehrsmittelwahl zum Ausdruck. Entsprechend dem NAM kennzeichnen personale Normen die persönliche moralische Verpflichtung zu einem prosozialen oder umweltschonenden Verhalten. Diese grenzen sich gegenüber sozialen Normen dadurch ab, dass man sich persönlich zu einem Verhalten moralisch verpflichtet fühlen kann, auch wenn dies von der umgebenden sozialen Bezugsgruppe anders erwartet wird. Im Bereich der Verkehrsmittelnutzung erweisen sich personale ökologische Normen vor allem hinsichtlich von umwelt- und klimaschutzbezogenen Aspekten als relevant. In vielen Studien lassen sich konsistent Zusammenhänge zwischen 
personalen Normen und der Nutzung alternativer Verkehrsmittel zum Pkw nachweisen (Harland, Staats \& Wilke, 1999; Lind, Nordfjærn, Jørgensen \& Rundmo, 2015).

Einstellungen kennzeichnen die subjektive Bewertung von Gegenständen, Personen und Situationen. Das Mobilitätsverhalten unterliegt hier ebenfalls subjektiven Bewertungen, die durch subjektive Wahrnehmungen und Präferenzen beeinflusst werden. So geht die Fortbewegung von A nach B im physikalischen Raum immer auch mit sozial vermittelten symbolischen Bewertungsprozessen seitens der Verkehrsteilnehmerinnen und Verkehrsteilnehmer einher (Jahn \& Wehling, 1999). Eine besondere Bedeutung kommt hierbei symbolisch und emotionalen Bewertungsprozessen unterschiedlicher Verkehrsmittel zu, die sich nicht hinreichend auf materiell-funktionale Aspekte, wie z. B. Zeit- und Kostenaufwand, zurückführen lassen (Steg, Vlek \& Slotergraf, 2001). Hunecke (2006) unterscheidet mit Blick auf die Verkehrsmittelnutzung vier symbolische Dimensionen: Autonomie, Erlebnis, Privatheit und Status, für die mehrfach Zusammenhänge zur Verkehrsmittelnutzung aufgezeigt worden sind (Hunecke, Haustein, Grischkat \& Böhler, 2007; Hunecke, Groth \& Wittowsky, 2020). Die symbolische Dimension Autonomie beschreibt, in welchem Maße Alltagsaktivitäten und die damit verbundene Raumüberwindung durch die eigene Verkehrsmittelnutzung realisiert werden können. Die Bewertung der Autonomie deckt sich in weiten Teilen mit dem handlungstheoretischen Konstrukt der wahrgenommenen Verhaltenskontrolle (PBC) aus der TPB. Die symbolische Dimension Erlebnis bezieht sich auf den hedonischen Wert bei der räumlichen Fortbewegung und hängt stark davon ab, welche Erfahrungen dabei individuell als angenehm bewertet werden. Das können z.B. neue Eindrücke oder auch Ruhe und Entspannung sein. Die symbolische Dimension Status erfasst die gesellschaftliche Anerkennung, die mit der Nutzung von Verkehrsmitteln einhergeht. Die Bewertung des sozialen Status weist große inhaltliche Überschneidungen mit dem handlungstheoretischen Konstrukt der subjektiven Norm aus der TPB auf. Die symbolische Dimension der Privatheit kennzeichnet das allgemeine Bedürfnis nach einer selbstbestimmten Privatsphäre, die vor allem das Vermeiden von unerwünschten sozialen Kontakten in Verkehrsmitteln beinhaltet. Die Relevanz der vier symbolischen Mobilitätsdimensionen für die Verkehrsmittelnutzung variiert zwischen den Verkehrsmittelnutzerinnen und Verkehrsmittelnutzern und ist dabei sowohl von individuellen Präferenzen als auch von sozialkulturellen Unterschieden in der Bewertung der einzelnen Verkehrsmittel abhängig.

Kontrollüberzeugungen erfassen die subjektive Einschätzung von Personen, ihre anvisierten Verhaltensziele auch tatsächlich realisieren zu können. Auf die Verkehrs- mittelnutzung übertragen kennzeichnen Kontrollüberzeugungen subjektive Bewertungen der Umsetzbarkeit der eigenen Mobilitätsziele im jeweiligen Lebenskontext. In der Mobilitätspsychologie wurden Kontrollüberzeugungen am häufigsten über das handlungstheoretische Konstrukt der wahrgenommenen Verhaltenskontrolle (Perceived Behavior Control, PBC) aus der TPB erfasst. Neben der Intention erweist sich die PBC in der Regel als stärkster Einflussfaktor, wenn es um die Entscheidung geht, entweder den Pkw oder die Verkehrsmittel des Umweltverbundes zu nutzen (Lanzini \& Khan, 2017; Hoffmann et al., 2017).

In Analysen zur Verkehrsmittelnutzung (Haustein \& Hunecke, 2007; Haustein, Hunecke \& Manz. 2007) wurden zwei weitere verhaltensrelevante Kontrollüberzeugungen identifiziert: Wahrgenommene Mobilitätszwänge (Perceived Mobility Necessities, PMN) und die Rad-Wetterresistenz. PMN kennzeichnen das Ausmaß der eingeschätzten Notwendigkeit zur Umsetzung der Alltagsaktivitäten räumlich mobil sein zu müssen. Die PMN beziehen sich damit auf eine allgemeine Bewertung der Alltagsmobilität. Im Unterschied dazu wird die PBC in der Regel für eine spezifische Form der Verkehrsmittelnutzung erfasst, z. B. die ÖPNV- oder Radnutzung. Für die hier gewählte Konzeptualisierung der PMN, die sich auf verkehrsmittelübergreifende allgemeine Kontrollüberzeugungen zum eigenen Mobilitätsverhalten bezieht, konnte mit Hilfe von Strukturgleichungsmodellen ein unabhängiger Beitrag zur Erklärung der ÖV-Nutzung nachgewiesen werden (Haustein \& Hunecke, 2007). Während die mit der PBC erfassten Kontrollüberzeugungen hierbei stärker mit den Nutzungsmerkmalen des ÖV in Relation zur Pkw-Nutzung zusammenhängen, z. B. hinsichtlich von Kosten- und Aufwandsfaktoren, liegt der Fokus der PMN auf der allgemeinen Bewertung der Bedeutung der Mobilität für die eigene Alltagsorganisation. Als weitere Kontrollüberzeugung erfasst die Rad-Wetterresistenz die subjektive Überzeugung, das Rad auch bei schlechtem Wetter nutzen zu können. In einer Analyse von Mobilitätstagebüchern zeigte sich, dass Personen mit einer hohen Wetterresistenz zu allen Wetterlagen das Fahrrad bedeutend häufiger als Alltagsverkehrsmittel nutzen (Haustein et al., 2007).

Kontrollüberzeugungen werden in hohem Maße durch die subjektive Bewertung von objektivierbaren Merkmalen der räumlich-physikalischen Verkehrsinfrastruktur und von Erfordernissen der konkreten Alltagsorganisation bestimmt. Bei der Operationalisierung von Kontrollüberzeugungen gilt es zu entscheiden, welcher Auflösungsgrad für die zugrundeliegenden Nutzungsmerkmale gewählt wird. Hierbei können entweder jeweils Bewertungen spezifischer Nutzungsmerkmale von Verkehrsmitteln, z. B. die monetären Kosten oder die Erreichbarkeit, hinsichtlich ihrer Bedeutung für die eigene Person erfragt werden. Oder es wird ein Gesamturteil zur eigenen Handlungs- 
Tabelle 1. Stichprobendetails

\begin{tabular}{|c|c|c|c|c|c|c|c|c|c|}
\hline \multirow[t]{2}{*}{ Code } & \multirow[t]{2}{*}{ Jahr } & \multirow[t]{2}{*}{$N$} & \multicolumn{3}{|c|}{ Geschlecht } & \multicolumn{4}{|c|}{ Alter } \\
\hline & & & weiblich & männlich & $\mathrm{kA}$ & $\min$ & $\max$ & $M$ & $S D$ \\
\hline Studie I & 2003 & 1993 & 1056 & 937 & 0 & 18 & 81 & 46.7 & 16.6 \\
\hline Studie II & 2007 & 1001 & 584 & 417 & 0 & 16 & 88 & 40.3 & 14.0 \\
\hline Studie III & 2007 & 2003 & 1159 & 844 & 0 & 16 & 92 & 44.4 & 16.9 \\
\hline Studie IV & 2008 & 1343 & 822 & 521 & 0 & 14 & 87 & 51.0 & 15.8 \\
\hline Studie V & 2010 & 2513 & 1146 & 857 & 510 & 8 & 88 & 35.4 & 12.5 \\
\hline Studie VI & 2011 & 2000 & 841 & 1156 & 0 & 15 & 92 & 42.1 & 16.8 \\
\hline Gesamt & & 10850 & 5608 & 4732 & 510 & 8 & 92 & 43.2 & 16.4 \\
\hline
\end{tabular}

kontrolle erfasst, das auf einer zusammenfassenden Bewertung aller relevanten Kontrollüberzeugungen basiert. Zur Konzeptualisierung der beiden Konstrukte PBC und PMN wird in der vorliegenden Studie eine generalisierte Form von Kontrollüberzeugungen gewählt. Die Rad-Wetterresistenz erfasst hingegen eine spezifische Kontrollüberzeugung, die vor allem die Radnutzung maßgeblich beeinflusst.

\section{Ziel der Analyse}

Die vorliegende Arbeit hat das Ziel, auf der empirischen Grundlage von sechs Studien, die in einem Zeitraum von neun Jahren durchgeführt worden sind, (1) ein stabiles Messmodell für einen standardisierten Fragebogen zu psychologischen Einflussfaktoren der Verkehrsmittelnutzung (PsyVKN) zu generieren, (2) seine Güte und (3) die Zusammenhänge auf die selbstberichtete Verkehrsmittelnutzung zu prüfen.

\section{Methoden}

\section{Stichproben}

Die statistischen Analysen beruhen auf Daten aus insgesamt sechs Studien, in denen verschiedene Personengruppen im Zeitraum von 2003 bis 2011 untersucht worden sind. Die Gesamtstichprobe umfasst $N=10850$ Personen $($ weiblich $=5608$, männlich $=4$ 732, keine Angabe $=510)$ im Alter zwischen 8 und 92 Jahren $(M=43.16, S D=16.4)$. Altersspannen, Mittelwerte sowie Geschlechterverteilungen waren in den einzelnen Studien ähnlich (vgl. Tabelle 1).

Die Studien wurden in unterschiedlichen Zeiträumen mit unterschiedlichen Zielsetzungen sowohl zu wissenschaftlichen Forschungszwecken als auch im Rahmen der Marktforschung von Verkehrsunternehmen durchgeführt. Die Details sind für die vorliegende Analyse nicht rele- vant. Eine kurze Übersicht über die Projekte ist im Elektronischen Supplement 1 zu finden.

\section{Messinstrumente}

Der hier analysierte Fragebogen zu den psychologischen Einflussfaktoren der Nutzung von Pkw, ÖPNV und Fahrrad (PsyVKN) kam in allen Studien in nahezu identischer Form zum Einsatz. In vorangegangenen Studien (Hunecke, 2002) wurden ausgehend von den beiden Handlungstheorien TPB (Ajzen, 1991) und NAM (Schwartz, 1977) und den vier symbolischen Dimensionen der Mobilität, Autonomie, Erlebnis, Privatheit und Status (Hunecke, 2000) Items für die drei Hauptverkehrsmittel Pkw, ÖPNV und das Fahrrad entwickelt und getestet. Über die verschiedenen Studien hinweg wurden dabei sowohl einzelne Items in ihren Formulierungen optimiert bzw. entfernt als auch einzelne Konstrukte hinzugefügt. Die hier analysierte, abgeleitete Version des Instruments basiert auf den Items, die zum einen konsistent die psychologischen Konstrukte erfassen und sich zum anderen als Prädiktoren für die nahräumliche Verkehrsmittelnutzung empirisch bewährt haben.

Das Modell umfasst 8 Faktoren mit insgesamt 21 Items: Personale Ökologische Norm (Pnorm; 2 Items), Soziale Norm (Snorm; 2 Items), Wahrgenommene Verhaltenskontrolle (PBC; 4 Items), Wahrgenommene Mobilitätszwänge (PMN; 2 Items), Rad-Wetterresistenz (WetRes; 2 Items) und RadErlebnis (RadErl; 2 Items), Pkw-Orientierung (PkwOri; 5 Items) und Privatheit Öffentlicher Nahverkehr (PrvtOV; 2 Items). Alle Items sind in Tabelle 2 zu finden. Die acht Faktoren decken wesentliche Einflussfaktoren der Verkehrsmittelnutzung im Nahraum ab. Drei Faktoren beziehen sich auf die Nutzung des ÖPNV (Soziale Norm, Wahrgenommene Verhaltenskontrolle, Privatheit Öffentlicher Nahverkehr), zwei auf die Rad-Nutzung (Rad-Wetterresistenz, Rad-Erlebnis), ein Faktor auf die Pkw-Nutzung (Pkw-Orientierung) und zwei Faktoren auf verkehrs- 
mittelübergreifende Bewertungen (Personale Ökologische Norm, Wahrgenommene Mobilitätszwänge).

Die Items wurden in allen Studien auf einer 5-stufigen Skala erfasst. Die Formulierungen der in der vorliegenden Analyse betrachteten Items waren in allen Studien mit zwei Ausnahmen identisch, für die folgende Anpassungen vorgenommen wurden. Erstens wurde in Studie I anstelle eines Items des Faktors Personale Ökologische Norm (PNorm) ein später verworfenes, älteres Item der gleichen Skala verwendet (statt „Ich fühle mich verpflichtet, durch die Wahl meiner Verkehrsmittel einen Beitrag zum Klimaschutz zu leisten“ wurde „Aus ökologischen Gründen fühle ich mich verpflichtet, den Pkw im Alltag möglichst oft stehen zu lassen" verwendet). Zweitens musste in den Studien IV und V ein dort nicht verwendetes Item des Faktors Pkw-Orientierung durch multiple Imputation ergänzt werden („Ich schätze es, beim Autofahren selber darüber entscheiden zu können, mit welchen Personen ich zusammenfahren will“). Zuletzt wurden alle sonstigen fehlenden Einzelwerte als Missing at random identifiziert und per Multivariate Imputation by Chained Equations Verfahren aufgefüllt (Van Buuren \& GroothuisOudshoorn, 2011), sodass die Gesamtstichprobe in die Analysen mit einbezogen werden konnte.

Als abhängige Variablen zum Mobilitätsverhalten wurden aus den vorhandenen Daten der Studien I-V die selbst berichteten, relativen Anteile der Nutzung des Pkw, des öffentlichen Personennahverkehrs (ÖV) und des Fahrrades verwendet. Da diese Daten in fünf der sechs betrachteten Studien vorhanden waren, eigneten sich diese am besten für eine vergleichende Analyse.

\section{Statistische Analyse}

Zur Prüfung der Modellstruktur wurden konfirmatorisch Strukturgleichungsmodelle mit Mehrgruppenvergleich mit Maximum Likelihood Schätzung erstellt. Da Interkorrelationen zwischen den latenten Variablen anzunehmen waren, wurden keine orthogonalen Beziehungen vorgegeben.

Da die Studien aus Projekten mit deutlich unterschiedlichen Zielen, Kontexten und Schwerpunkten stammen und sehr unterschiedliche Stichprobenzusammensetzungen aufweisen, wurde eine Messinvarianz-Analyse (Byrne \& Watkins, 2003; Putnick \& Bornstein, 2016) durchgeführt, um die Vergleichbarkeit über die verschiedenen Studien hinweg zu prüfen und die Kontext- und Stichprobenunabhängigkeit des Instruments zu belegen. Aufgrund der hohen Fallzahl in allen Stichproben der Studien wurden die Modellfitindizes RMSEA, SRMR und insbesondere der CFI zur Beurteilung der Modellpassung genutzt. Es ist bekannt, dass $\chi^{2}$ als Kenngröße bei großen Stichproben die Passung systematisch überschätzt (Bollen, 1989).

Der CFI wurde genutzt, um bei der sequenziellen Messinvarianz-Analyse die Verschlechterung des Modellfits bei den systematischen Restriktionen zu beurteilen. Dabei wurde nach Cheung und Rensvold (2002) eine Verschlechterung um $\Delta$ CFI $>.01$ als kritisch beurteilt, woraufhin partielle Messinvarianz-Modelle generiert und geprüft wurden.

Um den Einfluss der Modelle auf Verkehrsmittelnutzung zu überprüfen, fungierte die relative Verkehrsmittelnutzung (Anteil der Pkw-Nutzung, Anteil öffentlicher Personennahverkehr (ÖV) und Anteil Fahrrad) in dem SEM als abhängige Variable. Studie VI konnte in diesem Schritt nicht berücksichtigt werden, weil darin keine äquivalente Variable erhoben wurde.

Zur Feststellung der diskriminanten Validität wurde eine Heterotrait-Monotrait Analyse (HTMT) durchgeführt (Henseler, Ringle \& Sarstedt, 2015). Zur Feststellung der konvergenten Validität wurden die durchschnittlich erklärten Varianzen (Average Variance Explained; AVE) für jede latente Variable geprüft, welche nach dem Fornell-Larcker-Kriterium $>.50$ ausfallen sollen (Fornell \& Larcker, 1981). Zur Reliabilitätsprüfung wurde neben dem Cronbachs Alpha auch die kongenerische Reliabilität (Compsite Reliability; CR) erfasst, die für Strukturgleichungsmodelle besser geeignet ist (Peterson \& Kim, 2013).

Die Berechnungen wurden mit R 3.6.2 (R Core Team, 2019) und insbesondere den packages lavaan (Rosseel, 2012), semTools (Jorgensen, Pornprasertmanit, Schoemann \& Rosseel, 2019) und psych (Revelle, 2019) durchgeführt.

\section{Ergebnisse}

\section{Strukturgleichungsmodelle}

Die Modellfitwerte der Strukturgleichungsmodelle für das 8-Faktoren-Modell waren $\chi^{2}(640)=4678.89, p<.001$; CFI $=.952$, RMSEA $=.046,90 \%$ CI [.045, .047], SRMR $=.039$, was guten bis sehr guten Fit-Werten entspricht (Hu \& Bentler, 1999).

\section{Item- und Skalenwerte}

Für Faktorladungen gibt es die Konvention, dass diese $\lambda=.30$ nicht unterschreiten sollten, wobei inhaltliche Erklärungen aber berücksichtigt werden sollten. Im getesteten Modell sind die Faktorladungen dementsprechend als zufriedenstellend einzuschätzen. Als einzige Abwei- 
Tabelle 2. Items und Itemkennwerte gemittelt über alle Studien

Skalen und Items (Kürzel)

8-Faktoren-Modell

$\begin{array}{llll}\lambda & \alpha & M & S D\end{array}$

Personale Ökologische Norm (PNorm)

Ich fühle mich aufgrund meiner Prinzipien persönlich verpflichtet, auf meinen Wegen im Alltag umweltfreundliche $\quad .85 \quad .71 \quad 2.95 \quad \begin{array}{ll}.34\end{array}$ Verkehrsmittel zu benutzen (PN1)

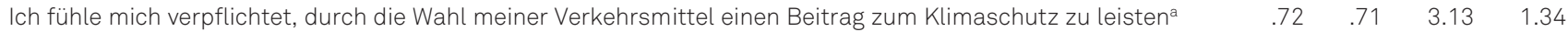
(PN3)

Soziale Norm (SNorm)

Menschen, die mir wichtig sind, denken, dass ich anstatt des Pkws öffentliche Verkehrsmittel nutzen sollte (SN1) $\quad .62 \quad \begin{array}{llll}.56 & 1.99 & 1.22\end{array}$

Menschen, die mir wichtig sind, würden mich unterstützen, wenn ich für meine Wege im Alltag anstatt des Pkws $\quad .69 \quad .56 \quad 2.47 \quad 1.36$ öffentliche Verkehrsmittel nutzen würde (SN2)

Wahrgenommene Verhaltenskontrolle (PBC)

Ich kann das, was ich tun will, mit öffentlichen Verkehrsmitteln erledigen (AutoOEV1)

Ich kann meinen Alltag sehr gut ohne Auto gestalten (AutoPkw2)

Für mich ist es schwer, die Wege in meinem Altag mit öffentlichen Verkehrsmitteln anstatt mit dem PKW

zurückzulegen (PBC1) (-)

Wenn ich will, ist es einfach für mich, öffentliche Verkehrsmittel anstatt des Pkws für meine Wege im Alltag $\quad \begin{array}{llll}.76 & .70 & 3.25 & 1.47\end{array}$ zu nutzen (PBC2)

Wahrgenommene Mobilitätszwänge (PMN)

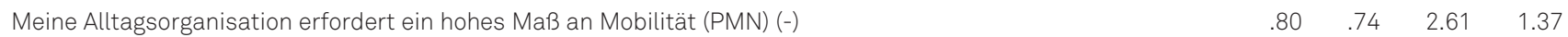

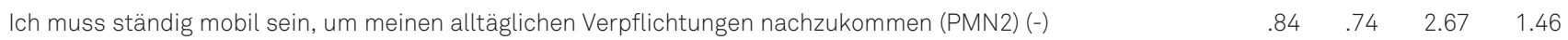

Rad-Wetterresistenz (WetRes)

Bei kühlem Wetter fahre ich ungern Rad (WetRes1) (-) $\quad .56 \quad .64 \quad 2.45 \quad 1.49$

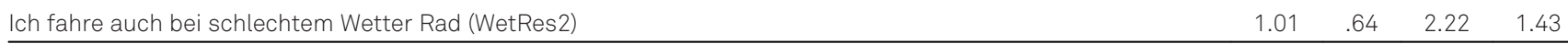

Radorientierung (RadErl)

$\begin{array}{lll}\text { Beim Radfahren kann ich mich gut entspannen (ErlRAD1) } & .75 \quad .78 \quad 3.44 & 1.44\end{array}$

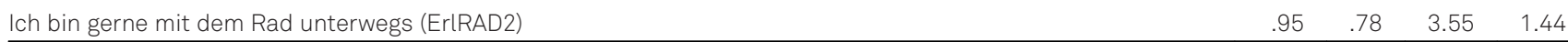

Pkw-Orientierung (PkwOri)

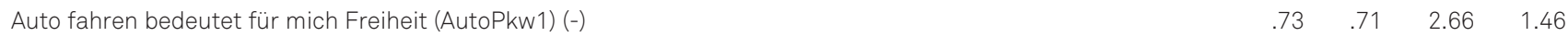

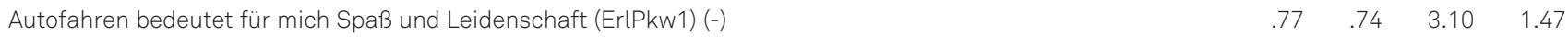

Mein fahrerisches Geschick beim Autofahren anwenden zu können, macht mir Spaß (ErlPKW2) (-) $\quad .69 \quad .67 \quad 3.31 \quad 1.48$

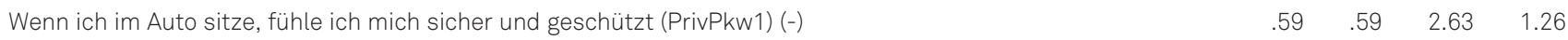

Ich schätze es, beim Auto fahren selber darüber entscheiden zu können, mit welchen Personen ich zusammen $\quad .50 \quad .49 \quad 2.58 \quad \begin{array}{lll}.45\end{array}$

fahren willb (PrivPkw2) (-)

Privatheit Öffentlicher Nahverkehr (PrvtOV)

In öffentlichen Verkehrsmitteln kommen mir Personen auf unangenehme Weise zu nahe (PrivOEV1) (-) $\quad .62 \quad .64 \quad 3.43 \quad 1.33$

In öffentlichen Verkehrsmitteln wird meine Privatsphäre auf unangenehme Weise eingeschränkt (PrivOEV2) (-) $\quad .85 \quad .64 \quad 3.64 \quad 1.24$

Anmerkungen: $\lambda$ = standardisierte Faktorladungen; $\alpha=$ Trennschärfe Cronbachs Alpha; $(-)=$ invertierte Items. ${ }^{a}$ in Studie I: "Aus ökologischen Gründen fühle ich mich verpflichtet, den Pkw im Alltag möglichst oft stehen zu lassen"; b für Studie IV und V imputiert.

chung findet sich eine Ladung von $\lambda=.22$ eines Items (WetRes1) auf die latente Variable Rad-Wetterresistenz in Studie VI. Alle Werte der SEM für alle Studien können den Diagrammen in den Abbildungen E1-E6 im Elektronischen Supplement 2 entnommen werden. Die gemittelten Itemkennwerte sind in Tabelle 2 dargestellt.

In Tabelle 3 sind die kongenerischen Reliabilitäten ( $\rho)$, Cronbachs Alpha $(\alpha)$ sowie die durchschnittlich erklärten Varianzen (AVE) und die Ergebnisse der HTMT Analyse zu finden. Die Reliabilitätsmaße bewegen sich im akzeptablen $(\rho>.70)$ bis guten $(\rho>.80)$ Bereich, fallen aber für die Soziale Norm gering aus $(\rho>.60)$. Zur Feststellung der konvergenten Validität sollten die AVE Werte mindestens $>.50$ betragen (Fornell \& Larcker, 1981). Dies traf in allen Fällen zu. Die Ergebniswerte der HTMT Analysen zur Feststellung der diskriminanten Validität sollten mindestens $<.85$ sein (Voorhees, Brady, Calantone \& Ramirez, 2016), was für alle Werte deutlich der Fall war. 
Tabelle 3. Kongenerische Reliabilität ( $\rho)$, Cronbachs $\alpha$, Durchschnittliche Erklärte Varianz (AVE) und HTMT Ergebnisse des 8-Faktoren-Modells

\begin{tabular}{|c|c|c|c|c|c|c|c|c|c|c|}
\hline \multirow[t]{2}{*}{ Skala } & \multirow[t]{2}{*}{$\rho$} & \multirow[t]{2}{*}{$\alpha$} & \multirow[t]{2}{*}{ AVE } & \multicolumn{7}{|c|}{ HTMT } \\
\hline & & & & A & B & $\mathrm{C}$ & $\mathrm{D}$ & $\mathrm{E}$ & $\mathrm{F}$ & $\mathrm{G}$ \\
\hline Personale Ökologische Norm (A) & .77 & .76 & .74 & & & & & & & \\
\hline Soziale Norm (B) & .60 & .60 & .61 & .47 & & & & & & \\
\hline Wahrgenommene Verhaltenskontrolle, PBC (C) & .83 & .82 & .63 & .32 & .39 & & & & & \\
\hline Wahrgenommene Mobilitätszwänge, PMN (D) & .80 & .79 & .75 & .04 & .04 & .47 & & & & \\
\hline Rad-Wetterresistenz (E) & .79 & .69 & .89 & .30 & .10 & .15 & .05 & & & \\
\hline Raderlebnis (F) & .84 & .83 & .84 & .31 & .06 & .08 & .03 & .58 & & \\
\hline Pkw-Orientierung (G) & .79 & .78 & .53 & .15 & .08 & .29 & .24 & .24 & .08 & \\
\hline Privatheit Öffentlicher Nahverkehr $(\mathrm{H})$ & .79 & .69 & .69 & .11 & .03 & .20 & .18 & .15 & .08 & .40 \\
\hline
\end{tabular}

Tabelle 4. Messinvarianzen für das 8-Faktorenmodell basierend auf 6 Studien

\begin{tabular}{|c|c|c|c|c|c|c|c|c|}
\hline Model & Modellvergleich & $\begin{array}{c}\chi^{2} \\
(d f)\end{array}$ & $\mathrm{CFI}$ & $\begin{array}{l}\text { RMSEA } \\
(90 \% \mathrm{Cl})\end{array}$ & SRMR & $\Delta \mathrm{CFI}$ & $\triangle \mathrm{RMSEA}$ & $\Delta \mathrm{SRMR}$ \\
\hline M1: Konfigural & - & $\begin{array}{c}4639.4^{* * *} \\
(966)\end{array}$ & .953 & $\begin{array}{c}.046 \\
(.045-.047)\end{array}$ & .039 & - & - & - \\
\hline M2: Metrisch & M2 vs M1 & $\begin{array}{c}5256.4^{\star \star *} \\
(1031)\end{array}$ & .946 & $\begin{array}{c}.048 \\
(.046-.049)\end{array}$ & .044 & .007 & .002 & .005 \\
\hline M3: Skalar & M3 vs M2 & $\begin{array}{c}7206.0 \text { *** } \\
(1096)\end{array}$ & .922 & $\begin{array}{c}.056 \\
(.054-.057)\end{array}$ & .049 & .024 & .008 & .005 \\
\hline M3a: Partiell Skalar & M3a vs M2 & $\begin{array}{c}6004.9 * * * \\
(1071)\end{array}$ & .937 & $\begin{array}{c}.050 \\
(.049-.052)\end{array}$ & .046 & .009 & .002 & .003 \\
\hline M4: Residual & M4 vs M3a & $\begin{array}{c}9019.2^{\text {*** }} \\
(1176)\end{array}$ & .899 & $\begin{array}{c}.061 \\
(.060-.062)\end{array}$ & .051 & .038 & .011 & .005 \\
\hline M4a: Partiell Residual & M4a vs M3a & $\begin{array}{c}6731.2^{* \star *} \\
(1121)\end{array}$ & .928 & $\begin{array}{c}.053 \\
(.051-.054)\end{array}$ & .049 & .009 & .002 & .002 \\
\hline
\end{tabular}

Anmerkungen: N = 10 850; Gruppen: Studie I N = 1993; Studie II N = 1 001; Studie III N = 2 003; Studie IV N = 1 343; Studie V N = 2513 ; Studie VI N = 2000 ; $\star p<.05,{ }^{*} p<.01,{ }^{* *} p<.001$.

\section{Messinvarianz über die sechs Studien}

Die Ergebnisse der Messinvarianz Analysen über die Studien sind in Tabelle 4 dargestellt. Entscheidend für das Akzeptanzurteil eines Restriktionsmodells war eine Verschlechterung des CFI um mehr als $\Delta$ CFI $>$.01. Es konnte volle konfigurale und volle metrische Invarianz sowie partielle skalare wie auch partielle residuale Invarianz festgestellt werden.

Zum Erreichen partieller skalarer Invarianz mussten beim entsprechenden Verfahrensschritt von den 21 Items die Intercepts von 5 Items von der Restriktion ausgenommen werden.

Zum Erreichen partieller residualer Invarianz mussten beim entsprechenden Verfahrensschritt von 21 Items die Residuen von 11 Items von der Restriktion ausgenommen werden.

\section{Regressionen der acht Modellfaktoren auf die selbstberichtete Verkehrsmittelnutzung}

Alle Ergebnisse der Regressionsanalysen auf Pkw, ÖV und Fahrrad sind im Detail im Elektronischen Supplement 3 zu finden. Tabelle 5 zeigt alle signifikanten, standardisierten Koeffizienten für die Faktoren.

Die PBC zeigte für alle Regressionen einen starken signifikanten Zusammenhang. Die anderen Variablen wiesen über alle Studien hinweg unterschiedliche Zusammenhänge auf. Die PMN, WetRes und PkwOri zeigten in jeder Studie signifikante Zusammenhänge mit den Variablen der Verkehrsmittelnutzung. Für die beiden Normen PNorm und SNorm sowie für die PrvtOV ließen sich jeweils unterschiedliche, jedoch mindestens in zwei Studien statistisch signifikante Zusammenhänge nachweisen. 
Tabelle 5. Übersicht der standardisierten Regressionskoeffizienten aus den SEM zu den Zusammenhängen zwischen den psychologischen Konstrukten des PsyVKN und den relativen Anteilen der Verkehrsmittelnutzung in den Studien I-V

\begin{tabular}{|c|c|c|c|c|c|c|c|c|c|}
\hline \multirow[t]{2}{*}{ VKN } & \multirow[t]{2}{*}{ St } & \multicolumn{8}{|c|}{ Standardized Regression Path Values } \\
\hline & & PNorm & SNorm & PBC & PMN & WetRes & RadErl & PkwOria & PrvtOV \\
\hline \multirow[t]{5}{*}{ MIV } & I & & & $-.601 * \star \star$ & & $-.183 * \star \star$ & & 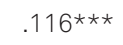 & \\
\hline & $\|$ & $-.111 \star \star \star$ & & $-.619 * \star \star$ & & $-.100 \star \star \star$ & & & \\
\hline & III & & & $-.553 * \star *$ & $-.064^{\star}$ & $-.141 * \star \star$ & & $.081 * * \star$ & $.047 *$ \\
\hline & IV & & & $-.663 * \star \star$ & & $-.061 \star \star$ & & $.133^{* * *}$ & $.070 * * *$ \\
\hline & V & & & $-.721 * \star \star *$ & & $-.139 * \star \star$ & & $.072 * \star \star$ & \\
\hline \multirow[t]{5}{*}{ OV } & 1 & & & $.555^{\star \star \star}$ & $-.204^{\star \star \star}$ & $-.080 \star \star$ & $-.094^{\star \star \star}$ & & \\
\hline & $\|$ & & & $.534^{\star \star \star}$ & $-.175^{\star \star \star}$ & & $-.120 * \star$ & & \\
\hline & III & & $.104 * \star \star$ & $.546^{\star \star \star}$ & $-.250 * \star \star$ & $-.178 * \star \star$ & $-.077 \star$ & $-.084 * \star$ & \\
\hline & IV & & & $.537 * \star \star$ & 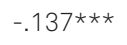 & $-.156 * \star \star$ & $-.086 * \star$ & $-.065 \star$ & $-.086 * \star$ \\
\hline & V & & & $.687 * * *$ & $-.184^{\star \star *}$ & $-.351 * \star *$ & & $-.084 * * *$ & \\
\hline \multirow[t]{5}{*}{ NMIV } & I & & & $.248^{\star \star \star}$ & 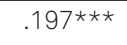 & $.268^{\star \star \star}$ & & $-.136 * \star \star$ & \\
\hline & II & $.103^{\star}$ & & $.276^{* \star *}$ & $.181 * \star \star$ & $.155^{\star \star \star}$ & $.120 * \star$ & & \\
\hline & III & & -.102 ** & $.066^{*}$ & 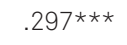 & $.309 * * *$ & & & \\
\hline & IV & & & $.214^{\star \star \star}$ & $.098^{\star \star}$ & $.256^{\star \star}$ & $.100 * \star$ & $-.088 * \star$ & \\
\hline & V & & & $.252^{\star * *}$ & $.123 * \star \star$ & $.446 * \star \star$ & $.067 *$ & & \\
\hline
\end{tabular}

Anmerkungen: St = Studie; VKN = Anteil genutzte Verkehrsmittel; MIV = Motorisierter Individualverkehr; OV = Öffentlicher Nahverkehr; NMIV = Nicht-motorisierter Individualverkehr; PNorm = Persönliche Norm; SNorm = Soziale Norm; PBC = Perceived Behavior Control; PMN = Perceived Mobility Necessity; WetRes = Rad-Wetterresistenz; RadErl = Rad Erlebnis; PKWOri = PKW Orientierung; PrvtOV = Privatheit Öffentliche Verkehrsmittel; * $p<.05, * \star p<.01$, $* \star * p<.001$ (n.s. nicht angegeben). ${ }^{a}$ invers erhobene Skalen zur besseren Nachvollziehbarkeit in der Tabelle reinvertiert.

\section{Diskussion}

Auf Grundlage der empirischen Daten aus sechs Studien mit 10850 Befragten konnte gezeigt werden, dass der PsyVKN in unterschiedlichen Stichproben unter verschiedenen Bedingungen ein reliables, valides und ökonomisches Messinstrument für psychologische Merkmale der Verkehrsmittelnutzung ist. Das Modell weist in den Strukturgleichungsmodellen sehr gute Fit-Werte und eine hohe Konstruktvalidität auf. Die Reliabilität ist für alle Konstrukte mit Ausnahme der Skala für die Soziale Norm zufriedenstellend. Ebenso konnte eine konfigurale und metrische Messinvarianz sowie partielle skalare und partielle residuale Messinvarianz über die verschiedenen Studien nachgewiesen werden, sodass ein Vergleich der Ergebnisse aus den sechs Studien möglich ist.

Die durchgeführten Regressionen demonstrieren, dass die Konstrukte des PsyVKN Zusammenhänge zur Verkehrsmittelnutzung zeigen und das Instrument deswegen eine sinnvolle Ergänzung zu Untersuchungen in der Mobilitätsforschung darstellt. Hierbei ist hervorzuheben, dass die Wahrgenommene Verhaltenskontrolle hinsichtlich des ÖV - in Übereinstimmung mit vorliegenden MetaAnalysen (Lanzini \& Khan, 2017; Hoffmann et al., 2017) den stärksten Einfluss vor allem auf die Nutzung öffentlicher Verkehrsmittel und dementsprechend die NichtNutzung des Pkws aufweist. Ein Grund dafür ist, dass sich
Kontrollüberzeugungen im Vergleich zu anderen psychologischen Konstrukten durch den stärksten Bezug zu objektivierbaren Merkmalen der vorhandenen Raum- und Verkehrsinfrastrukturen auszeichnen, die wiederum die Handlungsspielräume für die Verkehrsmittelnutzung wesentlich mitbestimmen. Schwächere, aber ebenfalls statistisch relevante Zusammenhänge zur Verkehrsmittelnutzung zeigen sich für die Personale Ökologische Norm und die Soziale Norm. Vor allem ökologische Verantwortungsnormen liefern für eine am Leitbild der nachhaltigen Entwicklung ausgerichtete Verkehrsplanung wichtige Informationen, wenn es um die Abschätzung der Akzeptanz der jeweils von den Planungsmaßnahmen betroffenen Zielgruppen geht. Die Berücksichtigung von verkehrsmittelspezifischen Einstellungen (Pkw-Orientierung, Rad-Erlebnis und Privatheit ÖV), die Bewertungen hinsichtlich der symbolischen Mobilitätsdimensionen Autonomie, Erlebnis und Privatheit beinhalten, empfiehlt sich vor allem für die Angebotsgestaltung von Verkehrs- und Mobilitätsanbietern.

Im PsyVKN wird für die drei Hauptverkehrsmittel Pkw, ÖV und Fahrrad eine unterschiedliche Anzahl von Einflussfaktoren erfasst. Die Identifizierung der ermittelten sechs verkehrsmittelspezifischen Konstrukte resultiert dabei nicht allein aus theoretischen Vorannahmen, sondern ist das Ergebnis der durchgeführten empirischen Analysen zur nahräumlichen Nutzung von Pkw, ÖV und 
Fahrrad. Hierbei zeigt sich konsistent, dass der ÖV differenziert hinsichtlich der Merkmale Autonomie, Privatheit und Soziale Norm bewertet wird. Die Bewertungen hinsichtlich des Pkw fallen hingegen in Bezug auf die drei theoretisch unterscheidbaren Mobilitätsdimensionen $\mathrm{Au}$ tonomie, Erlebnis und Privatheit so homogen aus, dass deren differenzierte Messung empirisch nicht zu rechtfertigen ist. Für das Rad lässt sich nur die Erlebnisdimension reliabel erfassen. Insgesamt deuten die acht identifizierten Konstrukte darauf hin, dass die Verkehrsmittelnutzung im Alltag im Wesentlichen auf Wahlentscheidungen zwischen den Verkehrsmitteln Pkw, ÖV und Fahrrad basiert. In keinem der sechs untersuchten Datensätze existieren empirische Hinweise, dass sich diese Bewertungsprozesse in einem psychologischen Konstrukt höherer Ordnung abbilden lassen.

Die unterschiedlichen Regressionskoeffizienten zwischen den sechs Studien verweisen auf eine unterschiedliche Bedeutung der ermittelten acht Konstrukte für die jeweils untersuchten Personengruppen und Raumtypen. Daher ist es nicht zuletzt auch aus ökonomischen Gründen sinnvoll, die für die jeweiligen Fragestellungen relevanten Skalen auszuwählen, um einzelne Konstrukte aus dem PsyVKN modular zu messen. Eine weitere Reduktion der ohnehin schon geringen Anzahl der Items pro Konstrukt ist dann jedoch nicht mehr möglich, ohne die Reliabilitäten der entsprechenden Skalen auf nicht mehr vertretbare Weise zu verringern. Nicht zuletzt wegen des geringen Umfanges wurden die Items des PsyVKN auch nach den hier berichteten Studien immer wieder zur Analyse mobilitätspsychologischer Fragestellungen eingesetzt (Von Behren et al., 2018; Hunecke et al., 2020; Groth, Hunecke \& Wittowski, 2021). Der PsyVKN besitzt das Potenzial, in standardisierten Verkehrserhebungen verwendet zu werden. Das betrifft auch die großen repräsentativen Verkehrserhebungen wie die MiD-Studie (Infas, 2018) oder das Mobilitätspanel (Ecke, Chlond, Magdolen, Eisenmann, Hilgert \& Vortisch, 2019), in denen bisher mobilitätsrelevante psychologische Aspekte mit dem Hinweis auf den hohen Aufwand für ihre Erhebung nicht oder nur sehr selektiv berücksichtigt wurden. Weiterhin eröffnet der PsyVKN Möglichkeiten, Fragestellungen innerhalb der interdisziplinären Verkehrs- und Mobilitätsforschung, beispielsweise hinsichtlich einer Abkehr vom privaten $\mathrm{Au}-$ tomobil, einer Renaissance des Fahrradfahrens oder der Multioptionalität bei der Verkehrsmittelnutzung, tiefergehend $\mathrm{zu}$ analysieren. Eine weitere Perspektive ergibt sich für das PsyVKN durch seine Übersetzung in andere Sprachen, die einen internationalen Einsatz ermöglichen. So wurde bereits ein Großteil der Items in die englische und chinesische (Magdolen, von Behren, Chlond, Hunecke \& Vortisch, 2019), die türkische und russische (Hunecke \& Ziesenitz, 2014) sowie die dänische Sprache (Haustein \&
Siren, 2014) übersetzt und in Mobilitätserhebungen erfolgreich eingesetzt. Die Überprüfung der Messinvarianz des PsyVKN für Versionen in unterschiedlichen Sprachen stellt jedoch eine Herausforderung dar, die erst noch in zukünftigen Analysen angegangen werden muss.

\section{Elektronische Supplemente (ESM)}

Die elektronischen Supplemente sind mit der OnlineVersion dieses Artikels verfügbar unter https://doi.org/ 10.1026/0012-1924/a000277

ESM 1. Projektbeschreibungen

ESM 2. SEM Modelle

ESM 3. Regressionen

\section{Literatur}

Ajzen, I. (1991). The theory of planned behavior. Organizational Behavior and Human Decision Processes, 50, 179-211. https:// doi.org/10.1016/0749-5978(91)90020-T

Bamberg, S., Ajzen, I. \& Schmidt, P. (2003). Choice of travel mode in the theory of planned behavior: The roles of past behavior, habit, and reasoned action. Basic and Applied Social Psychology, 25, 175-187. https://doi.org/10.1207/S15324834BASP 2503_01

Bamberg, S., Hunecke, M. \& Blöbaum, A. (2007). Social context, personal norms and the use of public transportation: Two field studies. Journal of Environmental Psychology, 27, 190-203. https://doi.org/10.1016/j.jenvp.2007.04.001

Bamberg, S. \& Schmidt, P. (2003). Incentives, morality, or habit? Predicting students' car use for university routes with the models of Ajzen, Schwartz, and Triandis. Environment and Behavior, 35, 264-285. https://doi.org/10.1177\%2F001391650225 0134

Bundesministerium für Verkehr und digitale Infrastruktur (Hrsg.) (2019). Verkehr in Zahlen 2019/2020. Flensburg: KraftfahrtBundesamt. Verfügbar unter: https://www.bmvi.de/SharedDocs/ DE/Publikationen/G/verkehr-in-zahlen-2019-pdf.pdf?__blob= publicationFile

Bollen, K. A. (1989). A new incremental fit index for general structural equation models. Sociological Methods \& Research, 17, 303 -316. https://doi.org/10.1177/0049124189017003004

Busch-Geertsema, A., Klinger, T. \& Lanzendorf, M. (2015). Wo bleibt eigentlich die Mobilitätspolitik? Eine kritische Auseinandersetzung mit Defiziten und Chancen der deutschen Politik und Forschung zu Verkehr und Mobilität. Informationen zur Raumentwicklung, 2, 135-148.

Byrne, B. M. \& Watkins, D. (2003). The issue of measurement invariance revisited. Journal of Cross-Cultural Psychology, 34, 155 - 175. https://doi.org/10.1177/0022022102250225

Cheung, G. W. \& Rensvold, R. B. (2002). Evaluating goodness-of-fit indexes for testing measurement invariance. Structural equation modeling, 9, 233 -255. https://doi.org/10.1207/S1532800 7SEM0902_5

Ecke, L., Chlond, B., Magdolen, M., Eisenmann, C., Hilgert, T. \& Vortisch, P. (2019). Deutsches Mobilitätspanel (MOP) - Wissenschaftliche Begleitung und Auswertungen, Bericht 2017/2018: 
Alltagsmobilität und Fahrleistung. Karlsruhe: Karlsruher Institut für Technologie (KIT). https://doi.org/10.5445/IR/1000091076

Fornell, C. \& Larcker, D. F. (1981). Evaluating structural equation models with unobservable variables and measurement error. Journal of Marketing Research, 18(1), 39-50. https://doi.org/ $10.2307 / 3151312$

Gardner, B. (2009). Modelling motivation and habit in stable travel mode contexts. Transportation Research Part F: Traffic Psychology and Behaviour,12(1), 68 - 76. https://doi.org/10.1016/j. trf.2008.08.001

Gardner, B. \& Abraham, C. (2008). Psychological correlates of car use: A meta-analysis. Transportation Research Part F: Traffic Psychology and Behaviour, 11, 300-311. https://doi.org/10. 1016/j.trf.2008.01.004

Groth, S., Hunecke, M. \& Wittowsky, D. (2021). Middle-Class, Cosmopolitans and Precariat among Millennials between Automobility and Multimodality. Transportation Research Interdisciplinary Perspectives, 12, 100467. https://doi.org/10.1016/j.trip. 2021.100467

Harland, P., Staats, H. \& Wilke, H. A. M. (1999). Explaining proenvironmental intention and behavior by personal norms and the theory of planned behavior. Journal of Applied Social Psychology, 29, 2505 -2528. https://doi.org/10.1111/j.1559-1816.1999. tb00123.x

Haustein, S. \& Hunecke, M. (2007). Reduced use of environmentally friendly modes of transportation caused by perceived mobility necessities: An extension of the theory of planned behavior. Journal of Applied Social Psychology, 37, 1856-1883. https://doi.org/10.1111/j.1559-1816.2007.00241.x

Haustein, S., Hunecke, M. \& Manz, W. (2007). Einfluss der Wetterlage und der Wetterempfindlichkeit auf die Verkehrsmittelnutzung. Internationales Verkehrswesen, 59, 392-396.

Haustein, S. \& Siren, A. (2014). Seniors' unmet mobility needs how important is a driving licence? Journal of Transport Geography, 41, 45-52. https://doi.org/10.1016/j.jtrangeo.2014.08. 001

Heath, Y. \& Gifford, R. (2002). Extending the theory of planned behavior: Predicting the use of public transportation. Journal of Applied Social Psychology, 32, $2154-2189$. https://doi.org/10. 1111/j.1559-1816.2002.tb02068.x

Henseler, J., Ringle, C.M. \& Sarstedt, M. A. (2015). New criterion for assessing discriminant validity in variance-based structural equation modeling. Journal of the Academy of Marketing Science, 43, 115 - 135. https://doi.org/10.1007/s11747-014-0403-8

Hoffmann, C., Abraham, C., White, M., Ball, S. \& Skippon, S. (2017). What cognitive mechanisms predict travel mode choice? A systematic review with meta-analysis. Transport Reviews, 37, 631 - 652. https://doi.org/10.1080/01441647.2017.1285819

Hu, L. \& Bentler, P.M. (1999). Cutoff criteria for fit indexes in covariance structure analysis: Conventional criteria versus new alternatives. Structural Equation Modeling: A Multidisciplinary Journal, 6(1), 1-55. https://doi.org/10.1080/10705519909540 118

Hunecke, M. (2000). Ökologische Verantwortung, Lebensstile und Umweltverhalten. Heidelberg: Asanger.

Hunecke, M. (2002). Umweltbewusstsein, symbolische Bewertung der Mobilität und Mobilitätsverhalten. In M. Hunecke, C. J. Tully \& D. Bäumer (Hrsg.), Mobilität von Jugendlichen. Psychologische, soziologische und umweltbezogene Ergebnisse und Gestaltungsempfehlungen (S. 47 -63). Wiesbaden: VS Verlag für Sozialwissenschaften. https://doi.org/10.1007/978-3-663-01176-7

Hunecke, M. (2006). Zwischen Wollen und Müssen: Ansatzpunkte zur Veränderung des Mobilitätsverhaltens. Zeitschrift für Technikfolgenabschätzung in Theorie und Praxis, 3(15), 31-37. https://doi.org/10.14512/tatup.15.3.31
Hunecke, M., Groth, S. \& Wittowsky, D. (2020). Young social milieus and multimodality: Interrelations of travel behaviours and psychographic characteristics. Mobilities, 15, 397-415. https:// doi.org/10.1080/17450101.2020.1732099

Hunecke, M., Haustein, S., Grischkat, S. \& Böhler, S. (2007). Psychological, sociodemographic, and infrastructural factors as determinants of ecological impact caused by mobility behavior. Journal of Environmental Psychology, 27, 277 - 292. https://doi. org/10.1016/j.jenvp.2007.08.001

Hunecke, M. \& Ziesenitz, A. (2014). Mobilitätsbezogene Einstellungen, Verhalten und $\mathrm{CO}_{2}$-Emissionen von russischsprachigen und türkeistämmigen Migrant_innen. In M. Hunecke \& A. Toprak (Hrsg.), Empowerment von Migrant_innen zum Klimaschutz (S. $51-76)$. München: oekom.

Institut für angewandte Sozialwissenschaft GmbH (2018). MiD Mobilität in Deutschland: Verkehrsaufkommen - Struktur Trends. Bonn: BMVI - Bundesministerium für Verkehr und Infrastruktur.

Jahn, T. \& Wehling, P. (1999). Das mehrdimensionale Mobilitätskonzept - Ein theoretischer Rahmen für die stadtökologische Mobilitätsforschung. In J. Friedrichs \& K. Hollaender (Hrsg.), Stadtökologische Forschung: Theorien und Anwendungen (S. $127-141)$. Berlin: Analytica.

Jorgensen, T. D., Pornprasertmanit, S., Schoemann, A. M. \& Rosseel, Y. (2019). semTools: Useful tools for structural equation modeling. R package version 0.5-2. Verfügbar unter: https:// CRAN.R-project.org/package $=$ semTools

Lanzini, P. \& Khan, S. A. (2017). Shedding light on the psychological and behavioral determinants of travel mode choice: A metaanalysis. Transportation Research Part F: Traffic Psychology and Behaviour, 48, 13-27. https://doi.org/10.1016/j.trf.2017. 04.020

Lind, H. B., Nordfjærn, T., Jørgensen, S. H. \& Rundmo, T. (2015). The value-belief-norm theory, personal norms and sustainable travel mode choice in urban areas. Journal of Environmental Psychology, 44, 119-125. https://doi.org/10.1016/j.jenvp.2015. 06.001

Lo, S. H., Van Breukelen, G. J. P., Peters, G.-J. Y. \& Kok, G. (2016). Commuting travel mode choice among office workers Comparing an Extended Theory of Planned Behavior model between regions and organizational sectors. Travel Behaviour and Society, 4, 1 - 10. https://doi.org/10.1016/j.tbs.2015.11.002

Magdolen, M., von Behren, S., Chlond, B., Hunecke, M. \& Vortisch, P. (2019). Combining attitudes and travel behavior - A comparison of urban mobility types identified in Shanghai, Berlin and San Francisco. Transportation Research Board 98th Annual Meeting. Washington, D.C. https://doi.org/10.5445/IR/1000093 783

Nordlund, A. \& Garvill, J. (2003). Effects of values, problem awareness, and personal norm on willingness to reduce personal car use. Journal of Environmental Psychology, 23, 339 - 347. https://doi.org/10.1016/S0272-4944(03)00037-9

Peterson, R. A. \& Kim, Y. (2013). On the relationship between coefficient alpha and composite reliability. Journal of Applied Psychology, 98, 194-198. https://doi.org/10.1037/a0030767

Putnick, D. L. \& Bornstein, M. H. (2016). Measurement invariance conventions and reporting: The state of the art and future directions for psychological research. Developmental Review, 41, 71 -90. https://doi.org/10.1016/j.dr.2016.06.004

R Core Team (2019). R: A language and environment for statistical computing. Wien: R Foundation for Statistical Computing.

Revelle, W. (2019). psych: Procedures for personality and psychological research. Illinois: Northwestern University. Verfügbar unter: https://CRAN.R-project.org/package=psych 
Rosseel, Y. (2012). lavaan: An R Package for Structural Equation Modeling. Journal of Statistical Software, 48(2), 1-36. http:// dx.doi.org/10.18637/jss.v048.i02

Scheiner, J. (2016). Verkehrsgeneseforschung: Wie entsteht Verkehr? In W. Canzler, A. Knie \& O. Schwedes (Hrsg.), Handbuch Verkehrspolitik (2. Aufl.) (S. 679-700). Wiesbaden: Springer VS.

Schwartz, S. H. (1977). Normative influences on altruism. Advances in Experimental Social Psychology, 10, 221-279. https:// doi.org/10.1016/S0065-2601(08)60358-5

Steg, L., Vlek, C. \& Slotegraaf, G. (2001). Instrumental-reasoned and symbolic-affective motives for using a motor car. Transportation Research Part F: Traffic Psychology and Behaviour, 4, 151 - 169. https://doi.org/10.1016/S1369-8478(01)00020-1

Van Buuren, S. \& Groothuis-Ouddshoorn, K. (2011). mice: Multivariate Imputation by Chained Equations in R. Journal of Statistical Software, 45(3), 1-67. http://dx.doi.org/10.18637/jss. v045.i03

Von Behren, S., Minster, C., Magdolen, M., Chlond, B., Hunecke, M. \& Vortisch, P. (2018). Bringing travel behavior and attitudes together: An integrated survey approach for clustering urban mobility types. 97th Transportation Research Board Annual Meeting, Washington, D.C. https://doi.org/10.5445/IR/1000093811
Voorhees, C. M., Brady, M. K., Calantone, R. \& Ramirez, E. (2016). Discriminant validity testing in marketing: An analysis, causes for concern, and proposed remedies. Journal of the Academy of Marketing Science, 44, 119-134. https://doi.org/10.1007/ s11747-015-0455-4

\section{Historie}

Onlineveröffentlichung: 19.11.2021

\section{Förderung}

Open Access-Veröffentlichung ermöglicht durch die Fachhochschule Dortmund

\section{Prof. Dr. Marcel Hunecke}

Fachbereich Angewandte Sozialwissenschaften

Fachhochschule Dortmund

Emil-Figge-Str. 44

44227 Dortmund

marcel.hunecke@fh-dortmund.de 